\title{
Cavitation regime detection through Proper Orthogonal Decomposition: dynamics analysis of the sheet cavity on a grooved convergent-divergent nozzle
}

\author{
A. Danlos ${ }^{\mathrm{a}, \mathrm{c}, *}$, F. Ravelet ${ }^{\mathrm{a}}$, O. Coutier-Delgosha ${ }^{\mathrm{b}}$, F. Bakir ${ }^{\mathrm{a}}$ \\ ${ }^{a}$ DynFluid Laboratory, EA 92, Arts et Métiers ParisTech \\ 151 Boulevard de l'Hôpital, 75013 Paris \\ ${ }^{b}$ Laboratoire de Mécanique de Lille, UMR 810\%, Arts et Métiers Paris Tech \\ 8 Boulevard Louis XIV, 59046 Lille \\ ${ }^{c}$ Laboratoire de Génie des Procédés pour l'Energie, l'Environnement et la Santé, EA 21, Conservatoire National des Arts et Métiers \\ 292 Rue Saint-Martin, 75003 Paris
}

\begin{abstract}
The unsteady character of the sheet cavity dynamics on the suction side of hydrofoils, on convergent-divergent nozzles or on blades in turbines and propellers is responsible for many issues like erosion, noise and vibrations. This two-phase flow dynamics is investigated using a robust method based on Proper Orthogonal Decomposition (POD). This method is applied to sequences of sheet cavity images, in order to identify the cavitation regimes (sheet cavity or cloud cavitation regimes). Once this method is validated on a reference case, POD calculation is used to evaluate the efficiency of a passive control method. Different longitudinal grooved surfaces are machined on the diverging wall of a Venturi. The grooves geometry allows to change the cavitation regime for a fixed cavitation number, and even to avoid the cloud cavitation shedding, which may damage structures.
\end{abstract}

Keywords: Proper Orthogonal Decomposition, Sheet cavity, Cloud cavitation, Passive control, Distributed organized roughness, Venturi-type section.

\section{Introduction}

Many methods are used to delay the cavitation inception, responsible for many issues like erosion, noise and vibrations. When pressure becomes inferior to the vapor pressure of the fluid, cavitation appears in the case of a flow over an obstacle, like hydrofoils, convergent-divergent nozzles or on blades in turbines and propellers (Mejri et al. (2006)). In some industrial configurations, it is difficult to avoid the cavitation inception. However, in some devices, cavitation can be an advantage for drag reduction or speed augmentation, especially in marine vehicles, by means of supercavitation (Ceccio (2010)). Moreover it is important to have a better knowledge of the two-phase flow dynamics once cavitation has appeared in order to reduce its negative effects and/or to increase its positive influence. A convergent-divergent step like a Venturi type section is a simple geometry that leads to a rich sheet cavitation dynamics (Barre et al. (2009); Campos-Amezcua et al. (2010)).

The development of the instability of a sheet cavity on a Venturi-type section has been widely studied (Stutz and Reboud (1997a,b); Callenaere et al. (2001); Dular et al. (2004)). Sheet cavity grows from the Venturi throat until a re-entrant jet appears in the sheet cavity closure, and

\footnotetext{
* Corresponding author

Email address: amelie.danlos@cnam.fr (A. Danlos)
}

flows upstream, near the wall, below the cavity. The reentrant jet then separates the sheet cavity when it reaches the liquid-gas interface of the flow. A large vapour cloud is shed and convected downstream, while the sheet cavity length is substantially reduced. Then, a cloud of vapor collapses in a higher pressure zone and sustains the re-entrant jet because of cloud implosion, which later produces a new shedding of vapor cloud (Callenaere et al. (2001); De Lange and De Bruin (1997)), that is characterized by the shedding frequency $f_{s}$.

Studies about the sheet cavity cycle observed on Venturi profiles have resulted in the definition of two types of oscillatory behaviors (Sayyaadi (2010)). A cloud cavitation regime appears when the sheet cavity is large enough and generates periodical shedding which generates vapor clouds before collapsing. When the sheet cavity length is shorter because of a higher pressure level and/or a Reynolds number smaller than a critical value (Keil et al. (2011, 2012)), no cloud cavitation shedding occurs. In this regime, the sheet cavity oscillates but the cavity closure position does not vary and no large scale cavitation structure shedding is observed. These two cavitation regimes are characterized by different values of the Strouhal number $S t_{L}=L f_{s} / v_{\text {throat }}$ where $L$ is the cavity length (defined in section 4.1 ) and $v_{\text {throat }}$ is the flow velocity measured at the Venturi throat. For a sheet cavity regime, $S t_{L} \simeq 0.1$ whereas a cloud cavitation regime is character-

February 3, 2014 
ized by $0.2 \leq S t_{L} \leq 0.4$ (Coutier-Delgosha et al. (2006); Dular et al. (2012)). The above thresholds are however not universal and may depend on the geometry or on the Reynolds number.

Furthermore, the cloud cavitation regime is more aggressive and leads to severe damages of solid structures. If a passive control method allows to reduce the sheet cavity length, it may then be possible to control the cavitation regime, in order to promote nearly stable regime instead of periodical unsteady behaviors. This can be done by modifying foil surface roughness. This technique has been used to delay cavitation inception, using local protuberances with different geometries or distributed irregularities (Arndt and Ippen (1968); Arndt (1981)). These modifications act on the turbulent boundary layer, which drives the onset of sheet cavitation. The role of surface condition has also been studied in a fully developed cavitating flow. Most of works about random distributed, transversal distributed, or local roughness show that it is possible to decrease sheet cavity length, in order to increase oscillation frequency of the cavity or even to change cavitation regime (Kawanami et al. (1997); Stutz (2003); Coutier-Delgosha et al. (2005); Yongjian et al. (2009)). But, to the best of our knowledge, there is no study about the effect of organized longitudinal roughness like grooves on the cavitation regime. This passive control method, applied on the suction side surface of a Venturi-type section with convergent and divergent angles of respectively $18^{\circ}$ and $8^{\circ}$ is discussed in this work.

In state-of-the-art studies, the different cavitation regimes are detected by observing the evolution of the sheet cavity development on acquired images. The lack of any rigorous, robust and efficient method to detect the change of cavitation regime handicaps studies about cavitation modeling or about passive control. In order to obtain a thorough analysis of the passive control effect on the sheet cavity dynamics, Proper Orthogonal Decomposition (POD) calculation (Berkooz et al. (1993)) is applied on images derived from fast visualization of the sheet cavity development. This method has already been applied on cavitating flows to characterize several differences between cavitation models (Utturkar et al. (2005a,b)) using multiscale differences of dynamics provided by each model. Other studies have shown the interest of the use of this type of fluid mechanics analysis tools for cavitating flows to better understand the sheet cavity cycle, with wavelets (Kjeldsen and Arndt (2001)) or POD and Dynamic Mode Decomposition (DMD) (Prothin et al. (2012)). After a description of the POD method and the advantages of its application on the sheet cavity regime study, results obtained with this rigorous analysis of the cloud cavitation shedding passive control with longitudinal grooves are presented to show the efficiency of this new cavitation regime detection technique.

\section{Proper Orthogonal Decomposition}

Proper Orthogonal Decomposition (POD) is a powerful tool to identify spatial and temporal coherent structures in a flow, providing a basis for a modal decomposition of a set of functions (Holmes P. and G. (1998)). In other domains, this procedure is named Karhunen-Loeve decomposition, Principal Components Analysis, Singular Systems Analysis or Singular Values Decomposition and finds applications in image processing, signal analysis, data compression, oceanography or fluid mechanics. Lumley (1967) was the first to transpose this technique in turbulence studies and to apply this post-processing on velocity fields (Berkooz et al. (1993)). Large scale structures, which contribute for a large part to the total kinetic energy of a velocity field, may then be highlighted and selected to reconstruct flow dynamics filtering stochastic events (PatteRouland et al. (2008)). By definition, POD is an optimal technique in an energetical sense that only a few eigenfunctions are necessary to recover flow dynamics. This linear process is based on spectrum theory of compact, self-adjoint operators. Eigenfunctions $\varphi$ are chosen in order to maximize the average projection of a data field $u$ on $\varphi$ :

$$
\max _{\varphi \in L^{2}([0,1])} \frac{\left\langle|(u, \varphi)|^{2}\right\rangle}{\|\varphi\|^{2}}
$$

where $|$.$| is the modulus, \langle$.$\rangle may be a spatial or a temporal$ averaging operation and $\|$.$\| is the L^{2}$-norm defined as:

$\|f\|=\left[\int_{0}^{1}|f(x)|^{2} d x\right]^{\frac{1}{2}}$

Maximization of $\left\langle|(u, \varphi)|^{2}\right\rangle$ with the constraint $\|\varphi\|^{2}=$ 1 leads up to the corresponding functional:

$$
J[\varphi]=\left\langle\|(u, \varphi)\|^{2}\right\rangle-\lambda\left(\|\varphi\|^{2}-1\right),
$$

which obeys for all variations $\varphi+\delta \psi$ to:

$$
\left.\frac{d}{d \delta} J[\varphi+\delta \psi]\right|_{\delta=0}=0 .
$$

This condition reduces to the integral equation:

$$
\int_{0}^{1}\left\langle u(x) u^{*}\left(x^{\prime}\right)\right\rangle \varphi\left(x^{\prime}\right) d x^{\prime}=\lambda \varphi(x) .
$$

The eigenfunctions $\left\{\varphi_{j}\right\}$ produce the optimal basis derived from Eq. (4) using the averaged autocorrelation function:

$$
R\left(x, x^{\prime}\right)=\left\langle u(x) u^{*}\left(x^{\prime}\right)\right\rangle .
$$

Using Hilbert-Schmidt theory, we can assume that there is an infinity of orthogonal eigenfunctions associated to eigenvalues obtained by the diagonal decomposition of the averaged autocorrelation function. As the averaged autocorrelation $R\left(x, x^{\prime}\right)$ is non-negative, eigenvalues $\lambda_{j}$ are ordered so that:

$$
\lambda_{j} \geq \lambda_{j+1} \geq 0
$$


Each data field $u$ may then be reconstructed from the modal decomposition based on the eigenfunctions $\varphi_{j}$ :

$$
u(x)=\sum_{j=1}^{\infty} a_{j} \varphi_{j}(x)
$$

where $a_{j}$ is a reconstruction coefficient defined as $\left\langle a_{j} a_{k}^{*}\right\rangle=\delta_{j k} \lambda_{j}$. If studied data fields are velocity fields, eigenvalues represent twice the average kinetic energy in each eigenmode $\varphi_{j}$. In this way, first modes, which are associated to the largest eigenvalues, refer to the most energetic structures of the flow field. POD is a method to extract disturbances in the flow at different energetic scales in the case of a turbulent velocity field application.

Here, POD is used to study sheet cavity dynamics by calculating the decomposition of sequences of images. In order to improve the calculation time, the snapshots method has been chosen (Sirovich (1987)). Indeed, in the classic method proposed by Lumley $(1967),\langle$.$\rangle is a tempo-$ ral average. $R\left(x, x^{\prime}\right)$ is then the spatial correlation tensor. In this case, when the $N$ fields $u$ present $M$ space points each with $n_{c}$ vector components, the size of the eigenvalues problem (4) is $N_{P O D}=M n_{c}$. But if $N<<M$, the snapshot methods allows to reduce the calculation time by using $\langle$.$\rangle as a spatial average, and the correlation tensor R$ is replaced by the temporal correlation tensor $C\left(t, t^{\prime}\right)$. In the snapshot method, the size of the eigenvalues problem becomes $N_{P O D}=N$.

\section{Experimental set-up}

Experiments were conducted in the closed loop test rig of the DynFluid laboratory water tunnel. Two storage tanks, with a capacity of $4 \mathrm{~m}^{3}$ each, provide water in the rig. The water flow is driven by a centrifugal pump. The test-section used for experiments is a $120 \times 100 \times 800 \mathrm{~mm}^{3}$ volume (Figure 1). A Venturi profile is placed in the test section with convergent and divergent angles respectively $18^{\circ}$ and $8^{\circ}$ because this configuration presents an unstable cavitation regime which is the most inconvenient case for industrial applications (Aeschlimann et al. (2013)). At the Venturi throat, the minimum height of the flow is $H_{\text {throat }}=67 \mathrm{~mm}$. All positions in the test section are expressed with non-dimensional values $x^{*}=x / H_{\text {throat }}$,

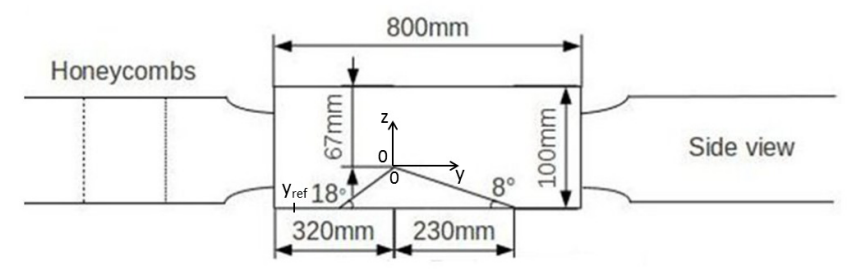

Figure 1: Test section of the experimental device using a Venturi profile $\left(H_{\text {throat }}=67 \mathrm{~mm}\right.$ is the reference length and $y_{\text {ref }}^{*}=$ $\left.y_{\text {ref }} / H_{\text {throat }}=-3.7\right)$.
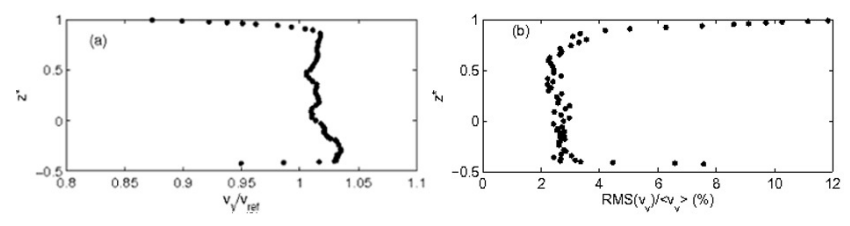

Figure 2: Characterization of the flow in the test section inlet in $y^{*} \simeq$ -4.5: (a) non-dimensional longitudinal velocity profile $v_{y}^{*}=v_{y} / v_{r e f}$ and $(b)$ the profile of the turbulence intensity $\frac{R M S\left(v_{y}\right)}{\left\langle v_{y}\right\rangle}$, where $\left\langle v_{y}\right\rangle$ is the time-averaged velocity.

$y^{*}=y / H_{\text {throat }}$ and $z^{*}=z / H_{\text {throat }}$. The flow rate is fixed for all measurements at $Q=(240 \pm 1) \mathrm{m}^{3} \cdot \mathrm{h}^{-1}$, which gives a discharge velocity at the Venturi throat $v_{\text {throat }} \simeq 8 \mathrm{~m} \cdot \mathrm{s}^{-1}$. Experiments are conducted at a temperature $T=(292 \pm 1) \mathrm{K}$. As the water viscosity in the operating conditions is $\nu=(1.04 \pm 0.03) 10^{-6} \mathrm{~m}^{2} . \mathrm{s}^{-1}$, the Reynolds number at the Venturi throat is $R e_{\text {throat }}=$ $v_{\text {throat }} H_{\text {throat }} / \nu=5.2 \times 10^{5} \pm 3 \%$. Several honeycombs and a circular contraction are used to obtain an almost homogeneous flow upstream of the obstacle.

A one dimensional Dantec Dynamics FlowExplorer Mini LDA system has been used to characterize this inlet flow by measuring the longitudinal velocity component $v_{y}$ of the flow. Laser Doppler Velocimetry (LDV) measurements have been conducted with 10000 recorded samples in a maximum time of acquisition of $60 \mathrm{~s}$ (with a data rate $300 \mathrm{~Hz}$ and a validation rate superior to 60\%). For all experimental configurations, at a distance $y=300 \mathrm{~mm}$ upstream of the Venturi throat (i.e. $4.5 H_{\text {throat }}$ upstream), the velocity profile is flat (spatially uniform) as we can see on Fig. 2(a). The turbulence intensity is less than $3 \%$ (Fig. 2(b)). A vacuum pump can decrease the pressure in the test rig by decreasing pressure at the free surface, in each storage tank, in order to act on the cavitation number $\sigma=\left(p_{\text {ref }}-p_{\text {vap }}\right) /\left(\frac{1}{2} \rho v_{\text {ref }}^{2}\right)$, with $p_{\text {ref }}$ the pressure measured at $y_{\text {ref }}=250 \mathrm{~mm}$ upstream of the Venturi throat (at $y_{\text {ref }}^{*}=y_{\text {ref }} / H_{\text {throat }} \simeq-3.73$ ) at the test section bottom, $v_{\text {ref }} \simeq 5.6 \mathrm{~m} . \mathrm{s}^{-1}$ the inlet discharge velocity measured in $y_{\text {ref }}$, and $p_{\text {vap }}$ the vapor pressure $\left(p_{\text {vap }} \simeq 2200 \mathrm{~Pa}\right.$ in operating conditions). For each studied configuration, $\sigma$ ranges from 0.9 to 1.7 with $\pm 1 \%$ of uncertainty.

Four plexiglas windows allow to make three-dimensional visualizations of the flow, in the test section, around the Venturi profile. In this study, only side vews of sheet cavi-

\begin{tabular}{|l|l|}
\hline Focal length & $100 \mathrm{~mm}$ \\
Resolution & $1280 \times 512$ pixels \\
Acquisition rate & $1000 \mathrm{fps}$ \\
Exposure time & $0.167 \mu \mathrm{s}$ \\
Pixels size & $12 \mu \mathrm{m} \times 12 \mu \mathrm{m}$ \\
Active area & $15.35 \mathrm{~mm} \times 12.29 \mathrm{~mm}$ \\
\hline
\end{tabular}

Table 1: Parameters of visualizations acquired with a CamRecord 600 Optronics camera and a Zeiss Makro-planar T* 

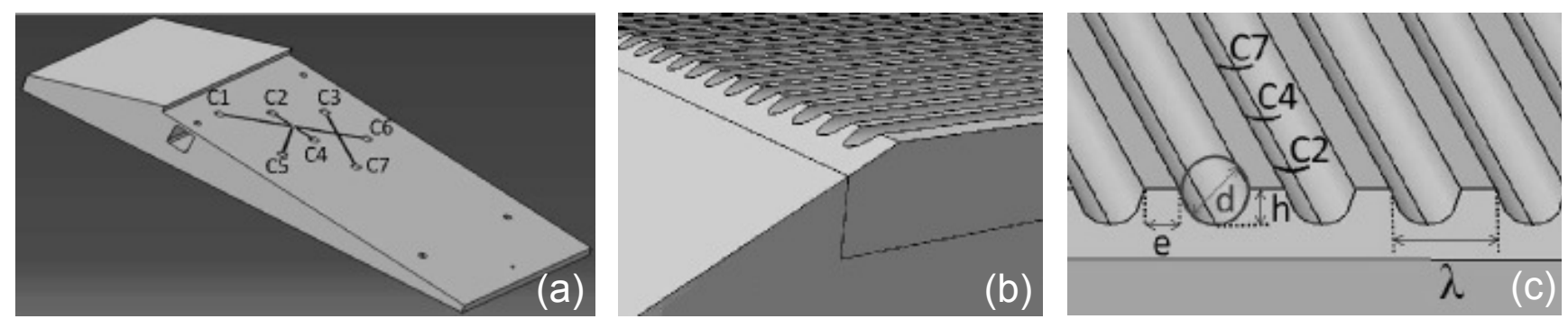

Figure 3: Venturi components: (a) Venturi basis with $C 1$ to $C 7$ pressure sensors on the basis surface (untapped in this study), (b) junction between the Venturi basis and a plate and $(c)$ zoom of a grooved plate with the definition of the grooves geometric parameters $(d$ and $h$ are respectively the grooves diameter and depth, $e$ is the width of the ridge and $\lambda$ is the grooves wavelength).

ties are acquired and analyzed. In order to visualize sheet cavity, 4000 images are acquired in each configuration. A CamRecord 600 Optronics camera with a Zeiss Makroplanar $\mathrm{T}^{*}$ objective lens is used. Parameters used for visualization measurements are presented in Table 1. The flow is illuminated in a continuous mode by a Super Long Life Ultra Bright (SLLUB) White Led Backlight from Phlox on a $200 \mathrm{~mm} \times 200 \mathrm{~mm}$ light output area. Its minimal luminance is $3000 \mathrm{~cd} . \mathrm{m}^{-2}$ and its uniformity is $99.54 \%$.

The bottom wall of the test section, in the divergent part of the Venturi, is made of $7 \mathrm{~mm}$ thick, $242 \mathrm{~mm}$ long, and $120 \mathrm{~mm}$ wide interchangeable brass plates, to cover the entire Venturi surface downstream of the Venturi throat as it is presented in figure 3 . The junction between the plate and the Venturi basis is done upstream of the Venturi throat to minimize disturbances on the flow. Plates are screwed on the basis and sealing between both components is ensured with vacuum grease. Table 2 presents the different grooved plates used for experiments. Plate A will be considered as reference, with a smooth surface on the suction side of the Venturi. Plates B to E are grooved surfaces made with a $d=1 \mathrm{~mm}$ or $2 \mathrm{~mm}$ diameter bur, with a depth $h$ and the width of the ridge is $e$. The distance between the middle of two adjacent grooves is equal to $\lambda=e+2 \sqrt{d h-h^{2}}$ for plates $B$ to $C$ and $\lambda=e+d$ for plates $D$ and $E$. Grooves open to the throat, so roughness affects the flow already from the onset of the sheet cavity.

\begin{tabular}{|l|c|c|c|c|c|ll|}
\hline & $d(\mathrm{~mm})$ & $h(\mathrm{~mm})$ & $e(\mathrm{~mm})$ & $\lambda(\mathrm{mm})$ & $N$ & $\begin{array}{l}\text { Symbols for } \\
\text { graphics }\end{array}$ \\
\hline$A$ & 0 & 0 & 0 & 0 & 0 & $\times$ & \\
$B$ & 1 & 0.25 & 0.1 & 0.97 & 124 & $\triangle$ & \\
$C$ & 2 & 1 & 0.1 & 2.1 & 57 & + & \\
$D$ & 2 & 2 & 1 & 3 & 40 & $\diamond$ & \\
$E$ & 2 & 2 & 0.1 & 2.1 & 57 & $\square$ & \\
\hline
\end{tabular}

Table 2: Characteristics of the studied plates used on the Venturi suction side

\section{Detection of the cavitation regime with POD calculation in the reference case of a smooth Venturi}

In order to highlight the efficiency of the POD method to detect the cavitation regime, the first step is to figure out the standard cavitation regime detection method. This method is described to analyze cavitation regimes obtained with the reference smooth case using post-processes cavitation images of a reference smooth plate. Then, these results are compared to POD analysis of the reference smooth case. The aim of this study is to explain the robustness of this efficient method.

\subsection{Standard method of cavitation images post-processing}

The cavitation regime of a sheet cavity developed on a Venturi profile can be determined by studying the Strouhal number associated with the variations of the closure position of the sheet cavity. This position is calculated with side view images of the sheet cavity, acquired with a camera.
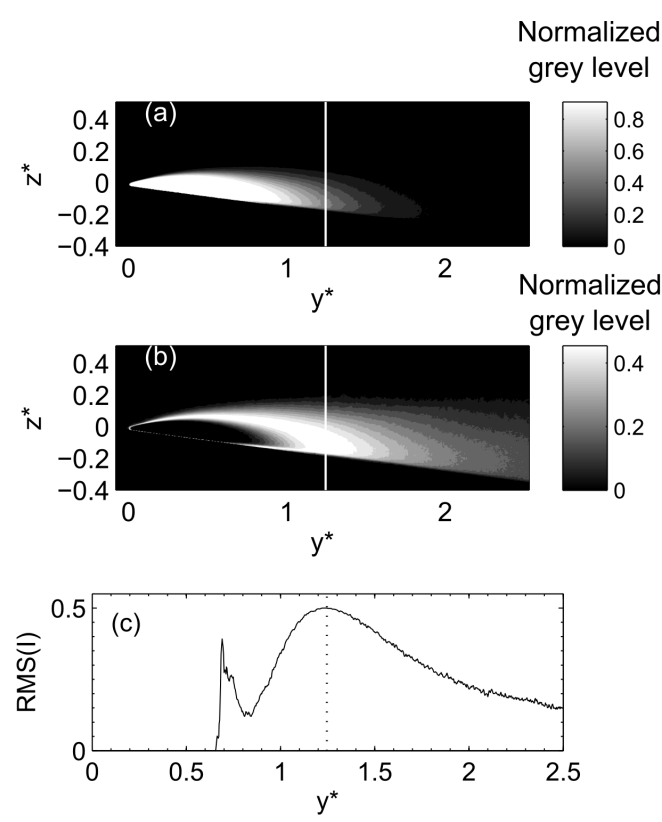

Figure 4: Sheet cavity on the smooth plate $A$ for $\sigma=1.18$ : (a) normalized average image, $(b)$ root mean square of normalized images (the line represents the non-dimensional sheet cavity length $L *=L / H_{\text {throat }} \simeq 1.62$ ) and $(c)$ a profile of the root mean square of normalized images $\operatorname{RMS}(I)$ plotted in $\mathrm{z}^{*}=0$.

Mean values and standard deviation of the grey levels of 4000 images in the $(y O z)$ plane (side views of the attached sheet cavity as shown in Fig. 1) are calculated. 
The first step is to normalize instantaneous images $I$ by a reference image $I_{0}$ (acquired with a non cavitating flow at rest). This normalization consists of the calculation of $\left(I-I_{0}\right) / I_{0}$. The closure of the sheet cavity is localized with the maximum value of the standard deviation of the grey levels calculated with the images stack (Dular et al. (2004)), as illustrated in figure 4. If we look at the profile of the root mean square of normalized images RMS(I) (figure $4 \mathrm{c}$ ), we can see a first peak in about $y *=0.7$. This value of $\mathrm{RMS}(\mathrm{I})$ corresponds to the vibration of the top part of the sheet cavity, close to the Venturi throat. At the beginning of the sheet cavity, grey levels oscillate between very low values (near 0 for the liquid phase) and very high values (near 1 for the vapor phase)(Dular et al. (2004)). Filters have been applied in the image processing to reduce this peak, in order to detect the maximum of RMS(I), corresponding to the closure position of the sheet cavity. The mean cavity length $L$ is then calculated from the Venturi throat to the closure line. The uncertainty of $L$, evaluated with the image processing by studying the effects of the threshold level for the binarization and filters applied on images is inferior to $2 \%$ (evaluated with the image processing by studying the effects of the threshold level for the binarization and filters applied on images). Considering the smooth plate $A$, mean cavity lengths obtained for different cavitation numbers $\sigma$ ranging from 1 to 1.9 with an uncertainty of $1 \%$ are presented in figure $5(\mathrm{a})$. When $\sigma$ decreases, the cavity length increases (Callenaere et al. (2001),Dular et al. (2012)).
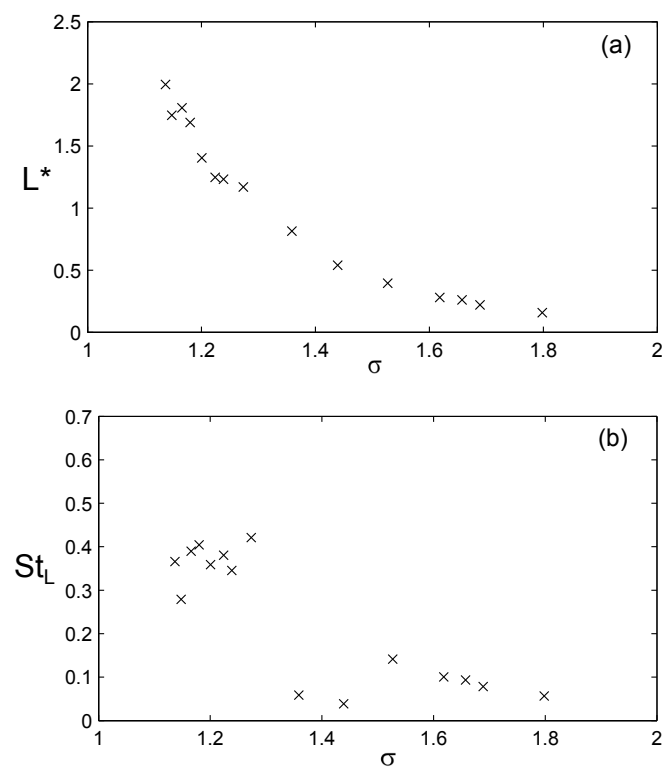

Figure 5: Standard post-processing images applied on the reference smooth plate $A$ : (a) non-dimensional sheet cavity length $L *=L / H_{\text {throat }}$ and (b) Strouhal numbers $S t_{L}=\frac{L f_{L}}{v_{\text {throat }}}$ according to the cavitation number $\sigma$

In order to study the different regimes of the sheet cavity, frequencies of sheet cavity oscillations have been determined using Fast Fourier Transform (FFT). A study of the grey levels at the sheet cavity closure position allows the calculation of the power spectral density associated with this phenomenon to determine the characteristic frequency of the sheet cavity. The uncertainty on the sheet cavity frequency is $0.2 \%$, according to convergence and images processing tests (number of images used, choice of grey level threshold...). Strouhal numbers associated to these sheet cavity frequencies are then calculated as $S t_{L}=L f_{L} / v_{\text {throat }}$, with $6 \%$ of uncertainty. Figure $5(\mathrm{~b})$ presents values of Strouhal numbers $S t_{L}$ for each studied plate, with respect to the cavitation number $\sigma$. Two different sheet cavity regimes can be identified. The first regime is called unstable regime and is defined by $0.2 \leqslant S t_{L} \leqslant 0.4$ which corresponds to a periodic cloud cavitation shedding, with large variations of the sheet cavity length. In the case of a smooth plate $A$, this cloud cavitation regime is encountered for $1 \leqslant \sigma \leqslant 1.4$. The second regime, called stable regime, is characterized by a small oscillation of the cavity closure, with an almost constant sheet cavity length and no convected cloud cavitation. Strouhal numbers in the stable regime are ranging from $0.05 \leqslant S t_{L} \leqslant 0.15$. This regime is obtained for $1.4 \leqslant \sigma \leqslant 2$ in the case of a smooth plate.

\subsection{POD post-processing of cavitation images}

POD is applied on normalized images of the side view of the sheet cavity. A convergence test allows to determine the number of snapshots necessary to validate the decomposition. Figure 6 presents the contribution of the first modes $j$ in the reconstruction of instantaneous images calculated as $\lambda_{j} / \sum_{j=1}^{N} \lambda_{j}$, from the eigenvalues $\lambda_{j}$ obtained by the decomposition with $N$ snapshots. This analysis shows that 600 snapshots are sufficient for the decomposition to obtain converged results.

As the optimal basis is given by the eigenfunctions $\varphi_{j}$ of Eq. (4), if $u(x)$ is a turbulent velocity field, the eigenvalues $\lambda_{j}$ represent twice the average kinetic energy in each mode $\varphi_{j}$. In this work, $u(x)$ is a grey level field and then, $\lambda_{j}$ is an information about the "weight" of modes in the reconstruction.

Figure 7 shows the first modes $\varphi_{j}$ (regardless the reconstruction coefficient $a_{j}$ ) obtained with POD of images in the smooth case study, for $\sigma=1.17$ and $\sigma=1.60$. These modes represent two different cavitation regimes according to the classic method of images post-processing (Fig. 5). As the sheet cavity length is small for $\sigma=1.60$, it is difficult to discuss this result, but we can see that contributions of each mode from modes 1 to 3 are smaller compared to the case with $\sigma=1.17$ (contributions are inferior to $0.3 \%$ for $\sigma=1.6$ and superior to $0.6 \%$ for $\sigma=1.17$ ). In the case of a sheet cavity regime, without a cloud cavitation shedding, the first mode 0 is almost sufficient to reconstruct the sheet cavity dynamics.

To obtain the temporal behaviour of the sheet cavity and the cloud cavitation, we study phase portraits of the temporal amplitude $a_{j}$, corresponding to the reconstruction coefficient of the mode $\varphi_{j}$. Reconstruction coefficients $a_{2}$ 

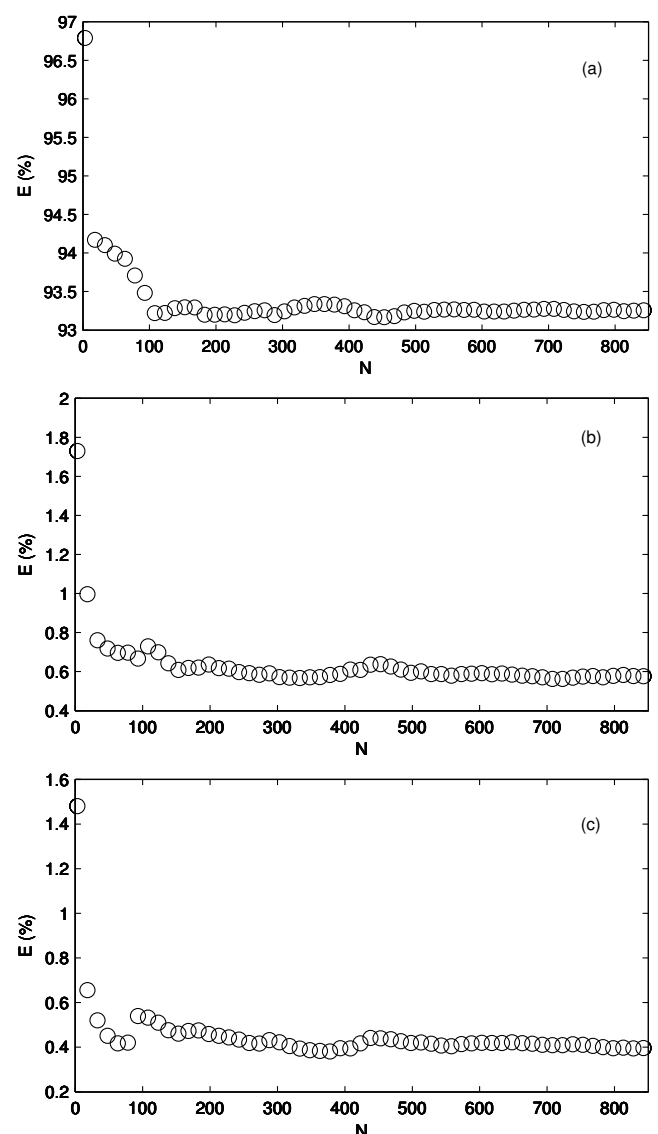

Figure 6: Convergence of the POD calculation for the plate $C$, with $\sigma=1.37$ : energy of modes $(a) 0,(b) 1$ and $(c) 2$ according to the number of images used for the POD

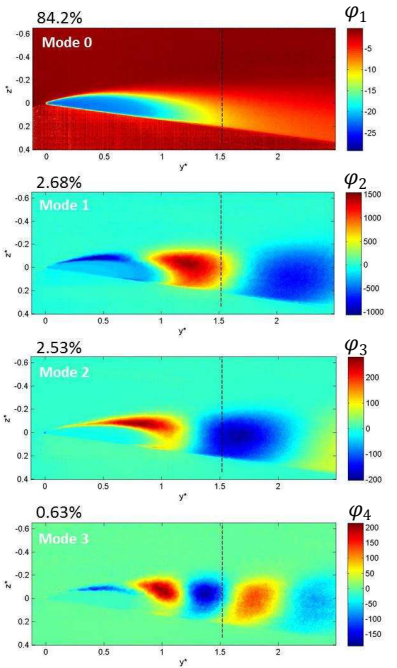

(a)

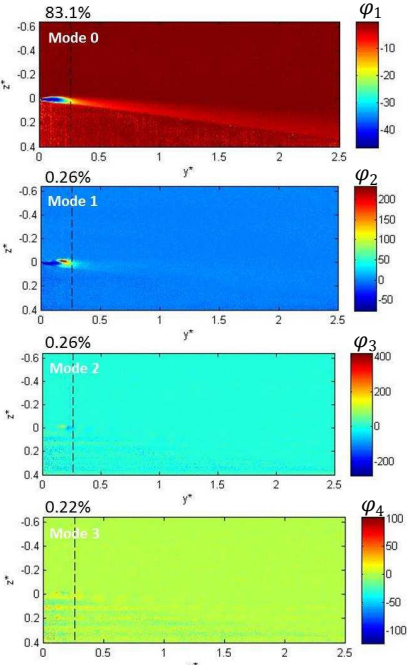

(b)
Figure 7: POD modes and their contribution in the reconstruction of instantaneous images (in percentages) for modes 0 to 3 , with the smooth plate $A$ : (a) for $\sigma=1.17\left(L^{*}=1.52\right)$ and (b)for $\sigma=1.60$ $\left(L^{*}=0.26\right)$
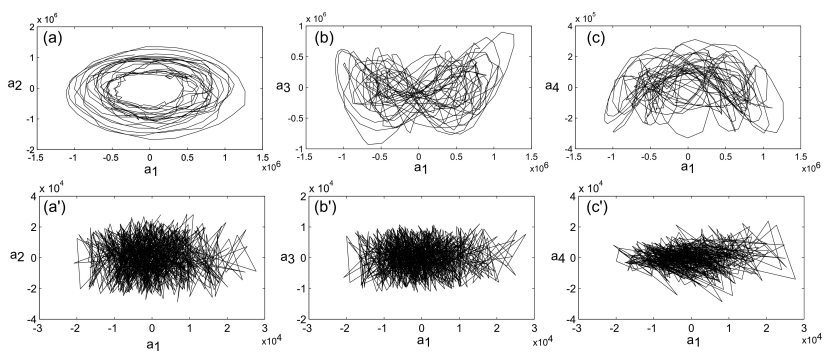

Figure 8: Phase portraits of POD reconstruction coefficients for modes 2 to 4 for the smooth plate $A$ : (a-c) with $\sigma=1.17$ and (a'-c') with $\sigma=1.60$

(mode 2 ) on figure $8(\mathrm{a})$ and coefficients $a_{3}$ (mode 3 ) on figure 8 (b) according to $a_{1}$ (mode 1 ) provide phase portraits similar to Lissajous figures (Oberleithner et al. (2011)) for $\sigma=1.17$. The circle described by data for figure $8(\mathrm{a})$ is characteristic of an oscillating phenomenon: two sine waves with equal frequencies and a 90 degrees phase shift give a perfect circle. Two sine waves in phase, with a frequency of the second wave twice the frequency of the first wave give a signal similar to figure $8(\mathrm{~b})$. These results explain figure $7(\mathrm{a})$ : modes 1 and 2 present phenomena with the same frequency but 90 degrees out-of-phase, while there are two frequencies between phenomena of modes 1 and 3 (with a frequency twice the other one) but a similar phase (Al-Khazali and Askari (2012)). On the other hand, for $\sigma=1.60$, results describe no precise drawing. It is a proof of the loss of periodicity provided by a cloud cavitation regime. On figure $8(\mathrm{~b})$, data describe another Lissajous figure which is characteristic of a periodical phenomenon, with two sine waves in phase but with different frequencies: the horizontal sine wave $\left(a_{1}\right)$ has a frequency twice the frequency of the sine wave $a_{3}$. The mode 3 is relative to the cloud cavitation in the case of $\sigma=1.17$. This result is very different from the sheet cavity regime presented for $\sigma=1.60$ on figure 8(b'). These phase portraits are then an efficient tool to detect the cavitation regime, using a robust mathematics method. 
5. Analysis of the longitudinal grooves effects on the cavity sheet developed on the divergent side of the Venturi

\subsection{Effects of the grooves on the sheet cavity closure po- sition}

In order to discuss the ability of longitudinal grooves to change the cavitation regime of the sheet cavity, the sheet cavity length is first determined in different operating conditions (grooves geometry or pressure conditions characterized by the cavitation number $\sigma$ ).

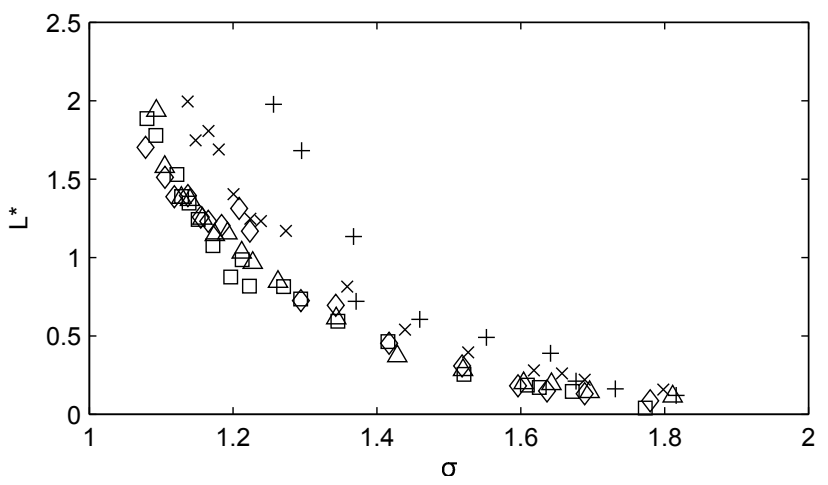

Figure 9: Non-dimensional sheet cavity length $L *=L / H_{\text {throat }}$ according to the cavitation number $\sigma: \times$ : plate $A, \triangle$ : plate $B,+$ : plate $C, \diamond$ : plate $D, \square$ : plate $E$.

Mean cavity lengths obtained for the different grooves geometries (using all plates presented in Table 2), and for cavitation number $\sigma$ ranging from 1 to 1.9 are presented in Figure 9. As we have seen previously, the mean cavity length decreases when the cavitation number increases and all grooved plates follow the same trend. The geometric parameters of different plates have effects on the mean cavity length for a fixed value of $\sigma$. For large grooves diameter $(d=2 \mathrm{~mm})$, the grooves depth depends is a crucial parameter: the comparison of results obtained for plates $C$ and $E$, which have same diameters, same spacing (number of grooves is $N=57$ for both cases) allows to measure the depth effect. For the plate $C$ with

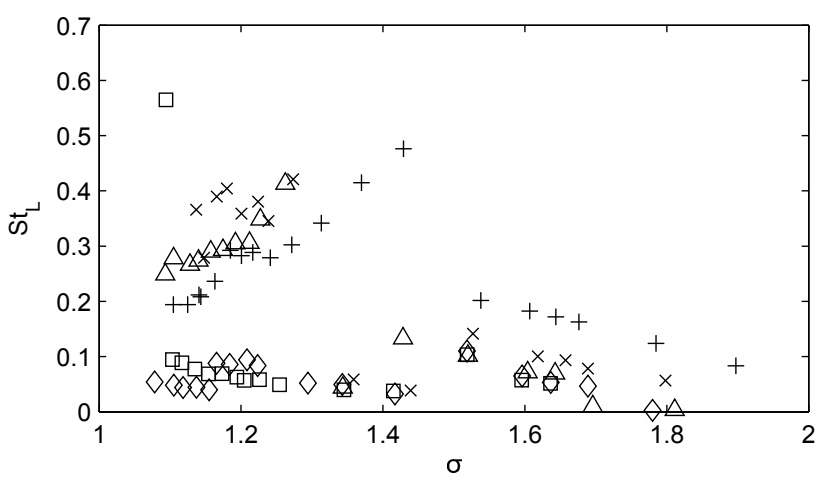

Figure 10: Strouhal number $S t_{L}=\frac{L f_{L}}{v_{\text {throat }}}$ according to the cavitation number $\sigma: \times$ : plate $A, \triangle$ : plate $B,+$ : plate $C, \diamond:$ plate $D, \square$ : plate E. $h=1 \mathrm{~mm}\left(h *=h / H_{\text {throat }} \simeq 0.016\right)$, the cavity mean length is larger than for other plates, for all $1 \leqslant \sigma \leqslant 1.9$. On the other hand, plate $E$ has a larger grooves depth $h=2 \mathrm{~mm}(h * \simeq 0.031)$ and presents smaller mean cavity lengths than the reference plate $A$. As we can see in literature, the cavity regime seems to depend on the mean length of the sheet cavity (Callenaere et al. (2001)), then it should be possible to modify the cavity regime by changing grooves geometry on the suction side of the Venturi-type section. Strouhal numbers, calculated for each grooved plate, are presented on Figure 10. For grooved plates, the range of cavitation numbers for each regime (defined with $0.2 \leqslant S t_{L} \leqslant 0.4$ for the unstable regime and with $0.05 \leqslant S t_{L} \leqslant 0.15$ for the stable regime) is similar to the smooth case, except for plates $D$ and $E$. For these two plates, the unstable regime is suppressed for the entire studied range $1 \leqslant \sigma \leqslant 1.8$ (see Media 1,2 proposed with Figure 11). Only one point for plate $E$ gives a Strouhal number $S t>0.5$. This Strouhal number is calculated with the frequency $f_{L}=44 \mathrm{~Hz}$, which is an harmonic of the sheet cavity oscillation frequency $f_{L}=9 \mathrm{~Hz}$. Then, this particular point corresponds also to a sheet cavity regime. 

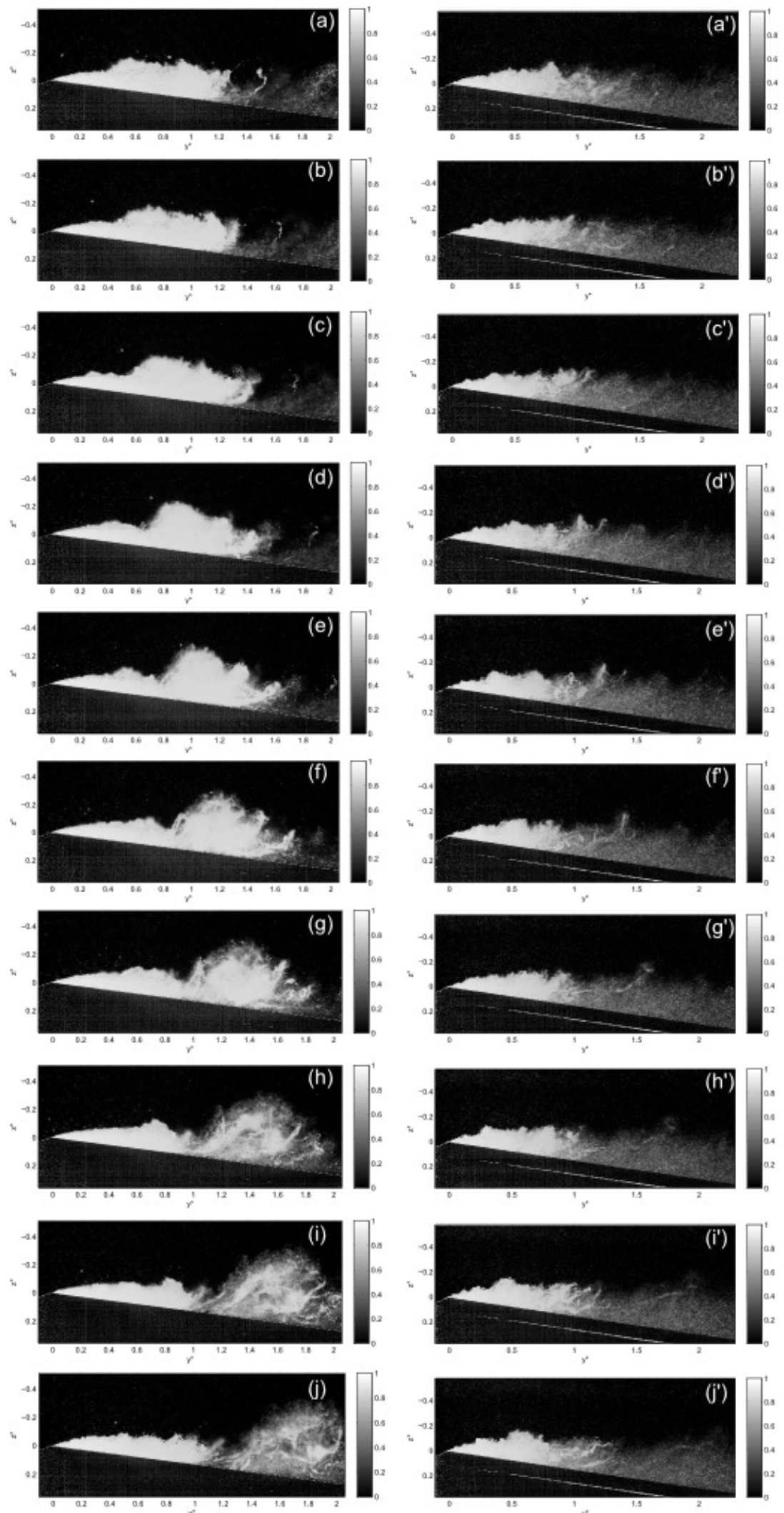

Figure 11: Visualisation of (a-j) the cloud cavitation shedding for the smooth plate $A$ and $\left(\mathrm{a}^{\prime}-\mathrm{j}{ }^{\prime}\right)$ the sheet cavity pulsation for the plate $E$, when $\sigma=1.17$ ( $\Delta t=2 \mathrm{~ms}$ between two images and colorbars represent normalized grey level). See Media 1-2 


\subsection{Dynamics analysis of the sheet cavity by POD}

POD is applied on 600 normalized images for the different grooved plates. Figure 12 shows the evolution of $\lambda_{j}$ according to modes, for different plates, with $\sigma=1.17$ in order to discuss the contribution of each mode in the reconstruction. Eigenvalues for the first mode 0 are $84.94 \%$ for the smooth case $A, 84.74 \%$ for plate $C, 74.27 \%$ for plate $B$ and $92.95 \%$ for plate $E$. For the other modes 1 to 50 , these eigenvalues are lower for the plate $E$ than for the other plates. This result highlights that for $\sigma=1.17$, the cavitation regime is different for plate $E$. As this regime is characterized only by a small variation of the closure position of the cavity sheet, the mode 0 is the most important in the reconstruction, in comparison with the other plates.

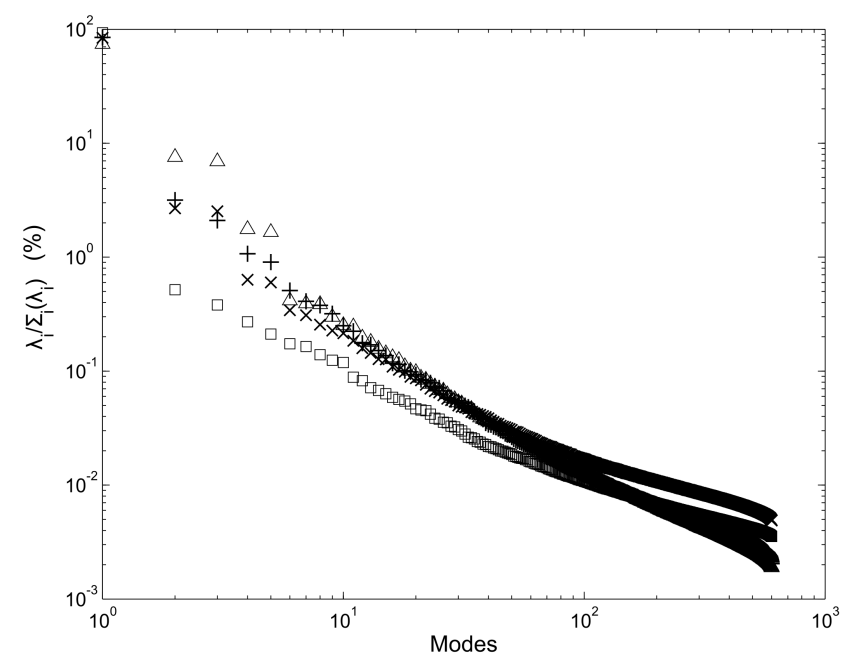

Figure 12: Contribution of POD modes in the reconstruction calculated with the eigenvalues $\frac{\lambda_{i}}{\Sigma_{i=1}^{N} \lambda_{i}}$, for $\sigma=1.17$ with $\times$ : plate $A, \triangle$ : plate $B,+$ : plate $C, \square$ : plate $E$.

First modes $\varphi_{j}$ are presented for plates $A, B, C$ and $E$ on figures 13 and 14 . Results for plate $D$ are not presented on these figures because they are very similar to plate $E$. As these figures present only modes, regardless the reconstruction coefficient $a_{j}$, the results interpretation does not allow to compare the order of magnitude of each mode in these different cases. We focus then on the position of vapor and liquid zones which are represented by different grey levels in the instantaneous images used for the decomposition, and on the global shape of modes. We can see that these first modes are quite similar for plates $A$ and $B$, even if the sheet cavity is shorter for the grooved plate $B$. The modes 0 to 3 represents $90 \%$ of the reconstruction of the instantaneous images for these two plates. For the plate $C$ which is characterized by a longer sheet cavity for the same cavitation number, modes are different. Only modes 0 to 2 are necessary to obtain $90 \%$ of the images reconstruction in this case. These modes are not very different from the first two cases, but from the mode 4 , the morphology of modes are different. Results obtained for the grooved plate $E$ show that the first mode
0 is sufficient to obtain $90 \%$ of the reconstruction. We can see that downstream of the closure position of the sheet cavity, there is no structure representing bubbles. This result confirms the previous analysis: no cloud cavitation is convected with plate $E$ for the entire range of studied cavitation number. The analysis of eigenmodes can then identify the type of cavitation regime, using the spatial behavior of the sheet cavity dynamics.

We then study phase portraits of the temporal amplitude $a_{j}$, corresponding to the reconstruction coefficient of the mode $\varphi_{j}$, as presented in figures 15 and 16. Reconstruction coefficients $a_{2}$ (mode 2 ) according to $a_{1}$ (mode 1) on figure 15 provide phase portraits similar to Lissajous figures (Oberleithner et al. (2011)) for plates $A$ and $B$ but not for plate $E$. This result shows again the efficiency of POD to detect the cavitation regime and then to measure the effect of a sheet cavity passive control.

Figure 16 presents phase portraits with the reconstruction coefficients $a_{3}$ for mode 3 according to $a_{1}$ for mode 1 . The mode 3 is relative to the cloud cavitation in this case (as it is explained in the section 4.2 with the analysis of the Lissajou's figures). We can see on figure 16 that the smooth plate $A$ and the grooved plate $B$ present a cloud cavitation regime. For the grooved plate $C$, the result is less characteristic of this regime than the smooth plate, in contrary to the result obtained previously with the analysis of the gray level variations. This information shows that the grooves depth or the wavelength of grooves acts on the sheet cavity dynamics $(\lambda>2 \mathrm{~mm}$ and $h>1 \mathrm{~mm}$ for plates $C, D$ and $E$ ). For plate $E$, the phase portrait $a_{3}=f\left(a_{1}\right)$ shows the sheet cavity regime.

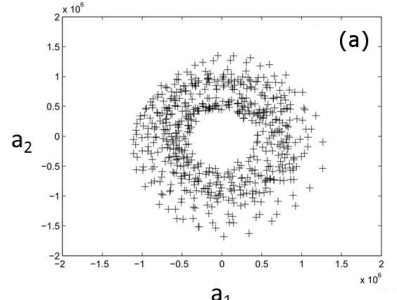

$a_{1}$

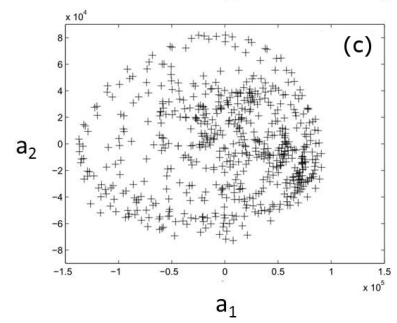

$a_{1}$

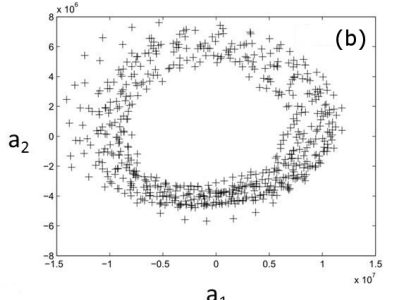

$a_{1}$

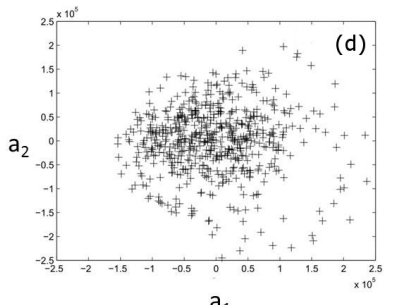

$a_{1}$
Figure 15: Phase portrait of POD reconstruction coefficients for modes 1 and 2, with $\sigma=1.17$ : (a) plate $A$, (b) plate $B$, (c) plate $C$ and (d) plate $E$.

We can see on Figures 17 (a) and (c) the temporal evolution of the reconstruction coefficient $a_{1}$ corresponding to the mode 1 , which is linked to the cloud cavitation, for plates $A$ and $E$ respectively. Results show the difference between these two plates: in the smooth case, the temporal 


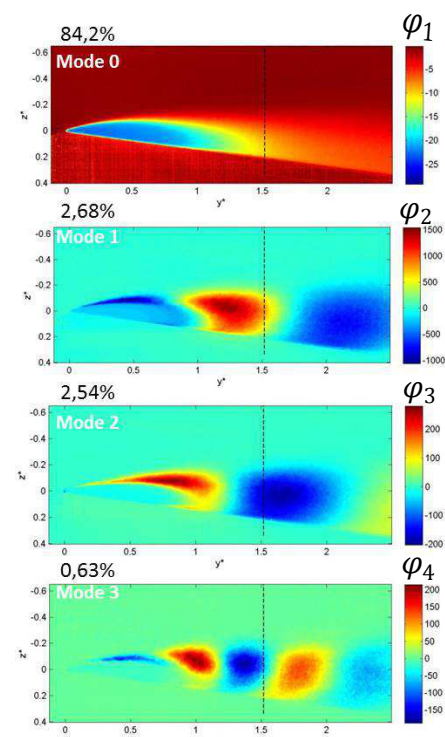

(a)

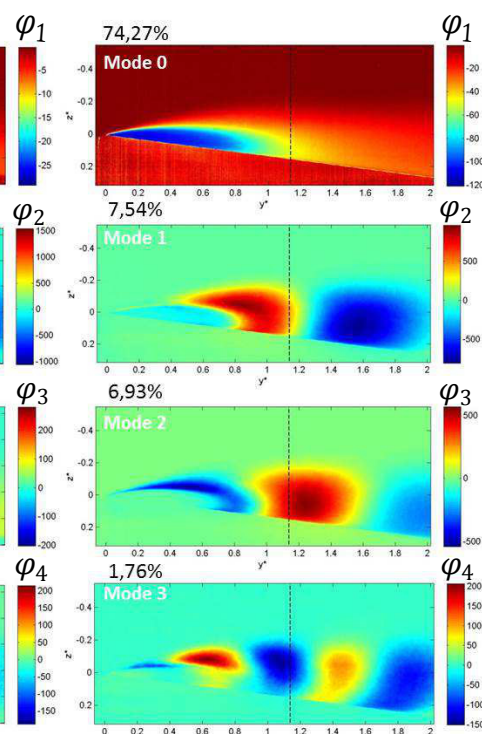

(b)

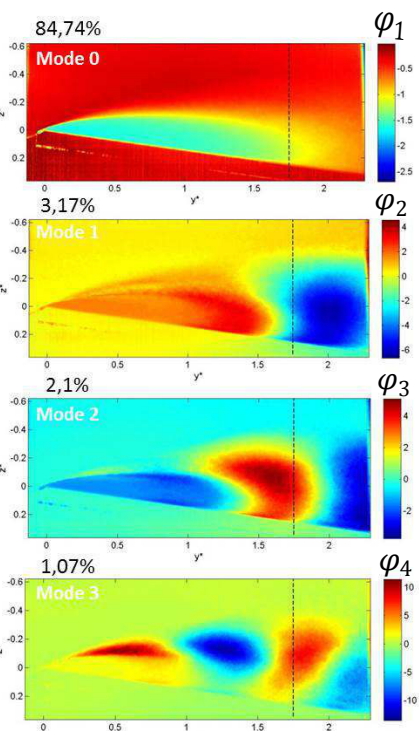

(c)

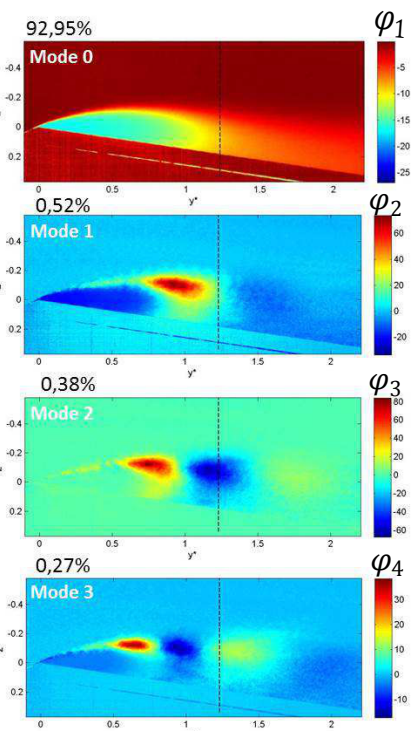

(d)

Figure 13: POD modes and their contribution in the reconstruction of instantaneous images (in percentages) for modes 0 to 3 , with $\sigma=1.17$ : (a) plate $A\left(L^{*}=1.52\right)$, (b) plate $B\left(L^{*}=1.14\right)$, (c) plate $C\left(L^{*}=1.75\right)$ and (d) plate $E\left(L^{*}=1.23\right)$.

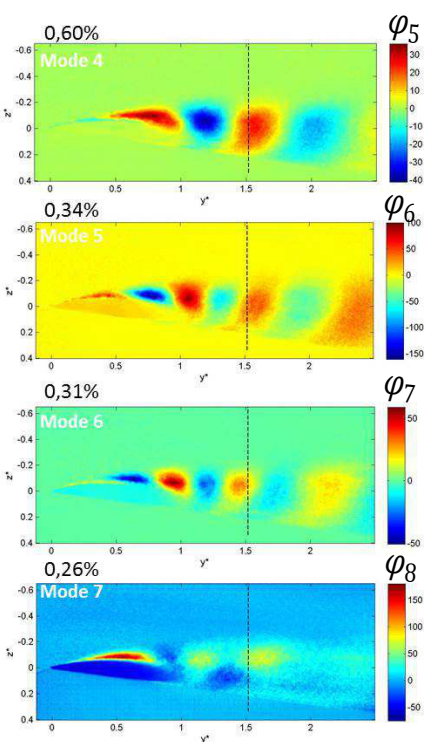

(a)

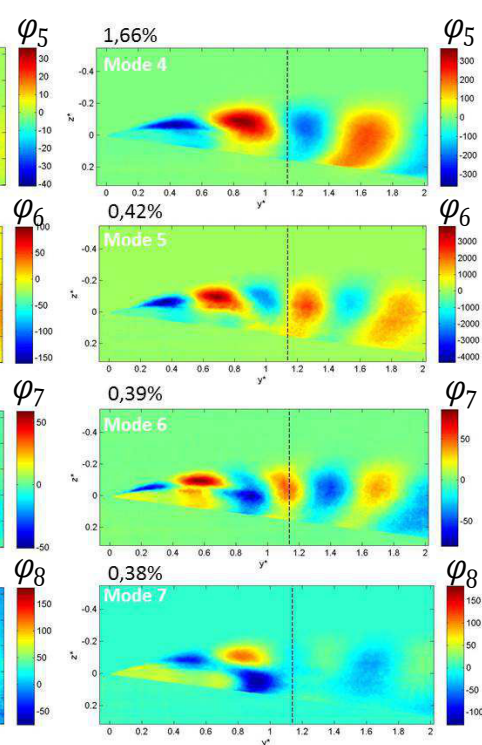

(b)

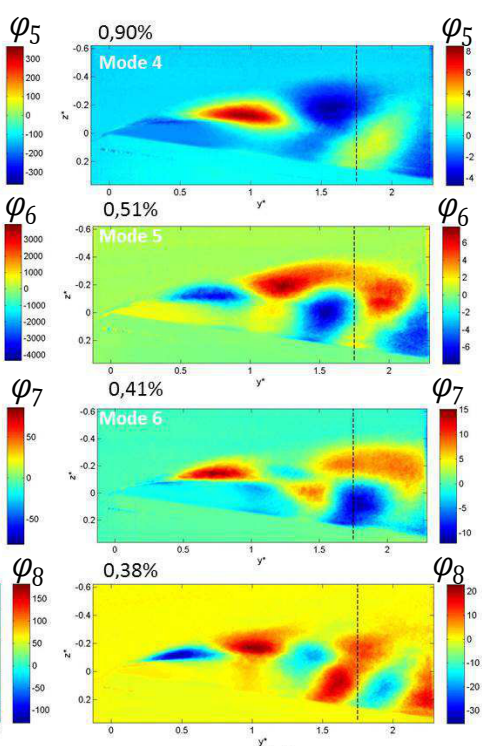

(c)

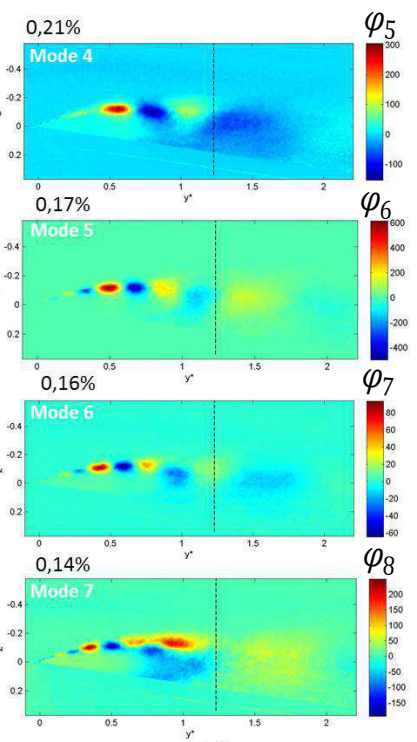

(d)

Figure 14: POD modes and their contribution in the reconstruction of instantaneous images (in percentages) for modes 4 to 7 , with $\sigma=1.17$ : (a) plate $A\left(L^{*}=1.52\right)$, (b) plate $B\left(L^{*}=1.14\right)$, (c) plate $C\left(L^{*}=1.75\right)$ and (d) plate $E\left(L^{*}=1.23\right)$. 

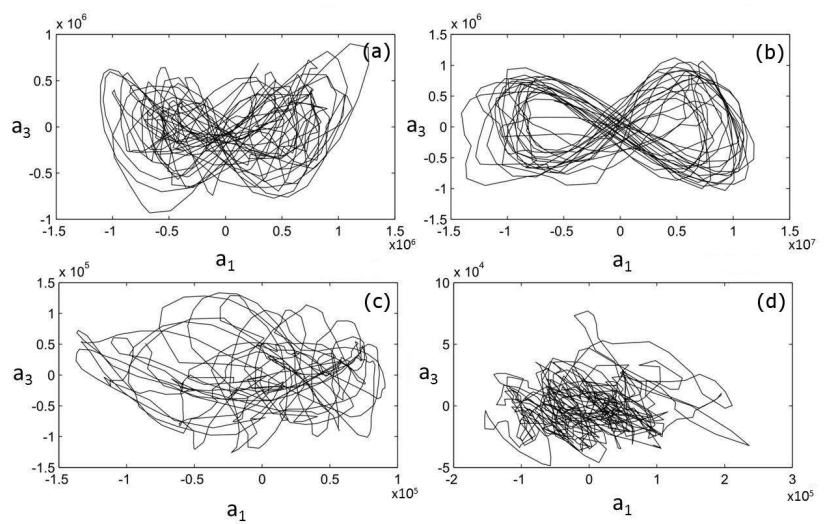

Figure 16: Phase portrait of POD reconstruction coefficients for modes 1 and 3, with $\sigma=1.17$ : (a) plate $A$, (b) plate $B$, (c) plate $C$ and (d) plate $E$.

evolution is periodic and similar to a sine wave, whereas the plate $E$ presents no periodic signal. Frequencies spectra calculated on these signals are presented on Figures 17 (b) and (d). The peak frequency for the plate $A$ is $35 \mathrm{~Hz}$ (with $1 \mathrm{~Hz}$ of uncertainty) while we have obtained a frequency $f_{L}=32 \mathrm{~Hz}$ by analyzing the images (section 5.1). For the plate $E$, the POD analysis gives a frequency of $8 \mathrm{~Hz}$ whereas the study of the images gives $9 \mathrm{~Hz}$ in the section 5.1. These results show that the POD analysis allows to detect the cloud cavitation or the sheet cavity regime but can also determine the frequency of the cloud cavitation shedding in the first case (as presented for the plate $A$ ) or the frequency of the sheet cavity oscillation in the sheet cavity regime, without cloud cavitation (about $8 \mathrm{~Hz}$ as seen for the plate $E$ ).
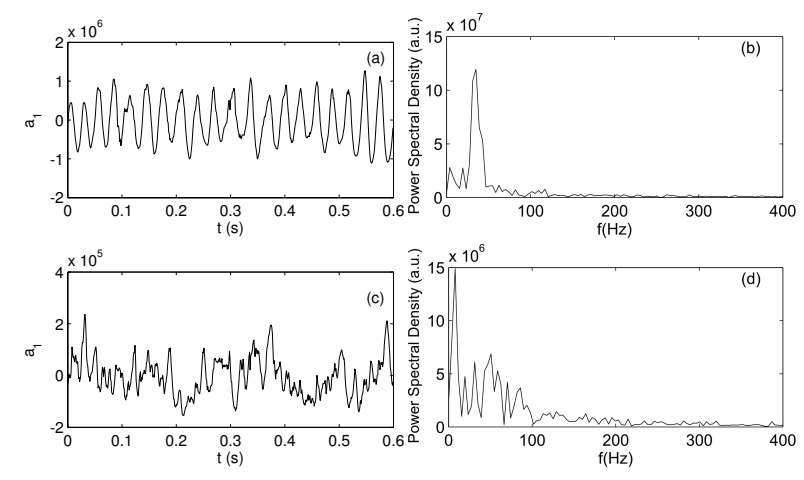

Figure 17: Reconstruction coefficient $a_{1}$ of the mode 1: (a) temporal evolution for plate $A$, (b) frequencies spectrum for plate $A$, (c) temporal evolution for plate $E$ and (d) frequencies spectrum for plate E.

\section{Conclusion}

Side view images of the sheet cavity dynamics on a converging-diverging profile have been analyzed with a POD method, in order to detect the effects of grooved surfaces, with different geometries. Studying the reconstruction coefficients $a_{j}$ calculated with the eigenvalues $\lambda_{j}$, we can identify the sheet cavity regime with no cloud cavitation convected, which allows a reduction of surface erosion. Analyzing the energy content of the second mode 1 allows to detect this regime: in the case of a sheet cavity regime the energy content of mode 1 is lower than $1 \%$ (Figures $7(\mathrm{~b})$ and $13(\mathrm{~d})$ ), while this value is superior to $1 \%$ for a cloud cavitation regime (Figures $7(\mathrm{a}), 13(\mathrm{a}), 13(\mathrm{~b})$ and $13(\mathrm{c})$ ). This threshold value is not sufficient to obtain a robust method. However, the study of phase portraits allows to differentiate regimes with the observation of Lissajous figures. Then, this tool is efficient to characterize the cavitation regime but also to calculate the cloud cavitation shedding, avoiding the sheet cavity length calculation.

Proper Orthogonal Decomposition allows to study grooves effects. Results show that grooves with a large depth $(h>1 \mathrm{~mm})$ and a large wavelength $(\lambda>2 \mathrm{~mm})$ change the sheet cavity dynamics. For example, plates $D$ and $E(h=2 \mathrm{~mm}$ and $\lambda \geqslant 2.1 \mathrm{~mm})$ suppress the cloud cavitation: for the entire studied range of cavitation number $1 \leqslant \sigma \leqslant 1.8$ we obtain a sheet cavity regime, characterized by a Strouhal number $0.05 \leqslant S t_{L} \leqslant 0.15$. For the other plates, we can have a cloud cavitation regime, for $1 \leqslant \sigma \leqslant 1.3$, characterized by $0.2 \leqslant S t_{L} \leqslant 0.4$. As we can see with results obtained with different grooved plates, the cavitation regime is related to cavity length, but does not depend only on it (for examples plates $D$ and $E$ ). Avoiding cloud cavitation, which is the most aggressive phenomenon, can reduce erosion of surfaces. It is then interseted to modify the boundary layer with longitudinal grooves (with chosen values of depth and grooves wavelength). But this analysis is possible only with an efficient tool to identify the two cavitation regimes (stable or unstable regimes), based on a robust method, as presented in this study. In order to complete the analysis of the sheet cavity dynamics, it could be interesting to deepen these results with an analysis of top views of the sheet cavities on these grooved plates.

\section{References}

Aeschlimann, V., S., B., and H., D. (2013). Unsteady cavitation analysis using phase averaging and conditional approaches in a $2 \mathrm{~d}$ venturi flow. Open J. of Fluid Dynamics, 3:171-183.

Al-Khazali, H. and Askari, M. (2012). Geometrical and graphical representations analysis of lissajous figures in rotor dynamic system. IOSR J. of Engineering, 2(5):971-978.

Arndt, R. A. and Ippen, A. T. (1968). Rough surface effects on cavitation inception. J. of Basic Engineering, 90(2):249-261.

Arndt, R. E. A. (1981). Cavitation in fluid machinery and hydraulic structures. Annual Rev. of Fluid Mechanics, 13(1):273-326.

Barre, S., Rolland, J., Boitel, G., Goncalves, E., and Fortes Patella, R. (2009). Experiments and modeling of cavitating flows in venturi: attached sheet cavitation. European J. of Mechanics B/Fluids, 28(3):444 - 464 .

Berkooz, G., Holmes, P., and Lumley, J. (1993). The proper orthogonal decomposition in the analysis of turbulent flows. Annual Rev. of Fluid Mechanics, 25(1):539-575. 
Callenaere, M., Franc, J.-P., Michel, J.-M., and Riondet, M. (2001). The cavitation instability induced by the development of a reentrant jet. J. of Fluid Mechanics, 444:223-256.

Campos-Amezcua, R., Khelladi, S., Bakir, F., Mazur-Czerwiec, Z., Sarraf, C., and Rey, R. (2010). Numerical analysis of unsteady cavitating flow in an axial inducer. Proc. of the Institution of $\mathrm{Me}-$ chanical Engineers, Part A: J. of Power and Energy, 224(2):223238.

Ceccio, S. (2010). Friction drag reduction of external flows with bubble and gas injection. Annual Rev. of Fluid Mechanics, 42(1):183203.

Coutier-Delgosha, O., Devillers, J., Leriche, M., and Pichon, T. (2005). Effect of wall roughness on the dynamics of unsteady cavitation. J. of Fluids Engineering, 127(4):726-733.

Coutier-Delgosha, O., Devillers, J., Pichon, T., Vabre, A., Woo, R., and Legoupil, S. (2006). Internal structure and dynamics of sheet cavitation. Phys. of Fluids, 18(1):1-12.

De Lange, D. and De Bruin, G. (1997). Sheet cavitation and cloud cavitation, re-entrant jet and three-dimensionality. Appl. Scientific Res., 58(1-4):91-114.

Dular, M., Bachert, B., Stoffel, B., and Sirok, B. (2004). Relationship between cavitation structures and cavitation damage. Wear, 257(11):1176-1184.

Dular, M., Khlifa, I., Fuzier, S., Adama Maiga, M., and CoutierDelgosha, O. (2012). Scale effect on unsteady cloud cavitation. Experiments in Fluids, 53(5):1233-1250.

Holmes P., L. J. and G., B. (1998). Turbulence, Coherent Structures, Dynamical Systems and Symmetry. Cambridge University Press.

Kawanami, Y., Kato, H., Yamaguchi, H., Tagaya, Y., and Tanimura, M. (1997). Mechanism and control of cloud cavitation. J. of Fluids Engineering, 119(4):788-795.

Keil, T., Pelz, P., and Buttenbender, J. (2012). On the transition from sheet to cloud cavitation. In Proc. of the 8th International Symposium on Cavitation, Cav2012.

Keil, T., Pelz, P., Cordes, U., and Ludwig, G. (2011). Cloud cavitation and cavitation erosion in convergent divergent nozzle. In Proc. of WIRMC 3rd International Cavitation Forum.

Kjeldsen, M. and Arndt, R. (2001). Joint time frequency analysis techniques: a study of transitional dynamics in sheet/cloud cavitation. In Proc. of the International Symposium on Cavitation Cav2001.

Lumley, J. (1967). The structure of inhomogeneous turbulent flows. In A.M. Yaglom and V.I. Tatarski editors, Atmospheric Turbulence and Wave Propagation, Nauca, Moscow.

Mejri, I., Bakir, F., Rey, R., and Belamri, T. (2006). Comparison of computational results obtained from a homogeneous cavitation model with experimental investigations of three inducers. J. of Fluids Engineering, 128(6):1308-1323.

Oberleithner, K., Sieber, M., Nayeri, C. N., Paschereit, C. O., Petz, C., Hege, H.-C., Noack, B. R., and Wygnanski, I. (2011). Threedimensional coherent structures in a swirling jet undergoing vortex breakdown: stability analysis and empirical mode construction. J. of Fluid Mechanics, 679:383-414.

Patte-Rouland, B., Danlos, A., Lalizel, G., Rouland, E., and Paranthon, P. (2008). Proper orthogonal decomposition used for determination of the convection velocity of the initial zone of the annular jet. aerodynamic study and control of instabilities. J. of Fluid Dynamics, 1:1-10.

Prothin, S., Billard, J.-Y., and Djeridi, H. (2012). Traitement d'image utilisant la pod et la dmd pour l'étude du développement de la cavitation sur un naca0015. In Proc. des 13èmes Journées de l'Hydrodynamique.

Sayyaadi, H. (2010). Instability of the cavitating flow in a venturi reactor. Fluid Dynamics Res., 42(5):055503.

Sirovich, L. (1987). Method of snapshots. Quarterly of appl. mathematics, 45(3):561-571.

Stutz, B. (2003). Influence of roughness on the two phase flow structure of sheet cavitation. J. of Fluids Engineering, 125(4):652-659.

Stutz, B. and Reboud, J. L. (1997a). Experiments on unsteady cavitation. Experiments in Fluids, 22(3):191-198.

Stutz, B. and Reboud, J.-L. (1997b). Two-phase flow structure of sheet cavitation. Phys. of Fluids, 9(12):3678-3686.

Utturkar, Y., Wu, J., Wang, G., and Shyy, W. (2005a). Recent progress in modeling of cryogenic cavitation for liquid rocket propulsion. Prog. in Aerosp. Sciences, 41:558-608.

Utturkar, Y., Zhang, B., and Shyy, W. (2005b). Reduced-order description of fluid flow with moving boundaries by proper orthogonal decomposition. International J. of Heat and Fluid Flow, 26(2):276 - 288 .

Yongjian, L., Haosheng, C., Jiadao, W., and Darong, C. (2009). Effects of grooves on cavitation around the body of revolution. $J$. of Fluids Engineering, 132(1):1-7. 